\title{
Diffusing Coordination Risk
}

\author{
By Deepal Basak and Zhen Zhou
}

\section{Online Appendix}

\section{Cutoff Equilibrium}

Given a diffused policy $J$, a cutoff equilibrium specifies a threshold signal $\hat{s}_{j}$ for any $j=1,2 \ldots J$, such that an agent $i$ in group $j$ attacks the regime that passes the $j$ th viability test iff $s_{i}<\hat{s}_{j}$.

This implies that for any $\theta$, the aggregate attack from any group $j$ is $\alpha \mathbb{P}\left(s_{i}<\right.$ $\left.\hat{s}_{j} \mid \theta\right)=\alpha F\left(\sqrt{\tau}\left(\hat{s}_{j}-\theta\right)\right)$, which is decreasing in $\theta$. Define $\underline{\theta}_{j-1}$ such that if $\theta \geq \underline{\theta}_{j-1}$, then the regime will pass the $j$-th viability test. Conversely, if $\theta<\underline{\theta}_{j-1}$, then the regime will fail at or before group $j$. Then $\underline{\theta}_{j-1}$ is such that

$$
\underline{\theta}_{j-1}-\alpha \sum_{j^{\prime}=1}^{j-1} F\left(\sqrt{\tau}\left(\hat{s}_{j^{\prime}}-\underline{\theta}_{j-1}\right)\right)=0 .
$$

Note that $\underline{\theta}_{0}=0$ and define

$$
\underline{\theta}_{J}=\hat{\theta}
$$

This means when $\theta=\hat{\theta}$, the regime has the exact strength to meet the aggregate attack from all the groups. Hence, any regime with $\theta \geq \hat{\theta}$ will survive.

Since viability tests remove the lower dominance region, it is always possible that in equilibrium the agents stop attacking a viable regime after the $j$-th test, for some $j=1,2, \ldots, J$. Suppose that in equilibrium the agents attack a viable regime until group $\bar{j} \leq J$, for some $\bar{j} \cdot \bar{j}=0$ means no agent attacks the regime. If $\bar{j}>0$, then $\underline{\theta}_{j-1}$ does not change after $\bar{j}$, i.e., once the regime survives the attack from the first $\bar{j}$ groups, it survives in the end. Then, any agent $i$ in group $j \leq \bar{j}$ who receives the cutoff signal $\hat{s}_{j}$ must be indifferent between attacking and not attacking - i.e.,

$$
\mathbb{P}\left(\theta \geq \hat{\theta} \mid \hat{s}_{j}, \theta \geq \underline{\theta}_{j-1}\right)=\frac{\int_{\hat{\theta}}^{\bar{\theta}} \pi(\theta) f\left(\sqrt{\tau}\left(\hat{s}_{j}-\theta\right)\right) d \theta}{\int_{\underline{\theta}_{j-1}}^{\bar{\theta}} \pi(\theta) f\left(\sqrt{\tau}\left(\hat{s}_{j}-\theta\right)\right) d \theta}=p .
$$

Thus, $\left\{\hat{s}_{j}\right\}_{j=1}^{J}$ constitutes a Perfect Bayesian Equilibrium in monotone strategies if there exists $\bar{j} \leq J$ such that 
1) for all $j>\bar{j}, \hat{s}_{j}=\underline{\theta}_{\bar{j}}-\frac{\sigma}{2}$ and consequently, it follows from Condition (PNV) that $\underline{\theta}_{\bar{j}}=\underline{\theta}_{\bar{j}+1}=\ldots=\underline{\theta}_{J}$

2) for all $j \leq \bar{j}, \hat{s}_{j}$ satisfies the indifference condition $\left(I^{v}\right)$, where the cutoffs $\left\{\underline{\theta}_{j-1}\right\}_{j=1}^{J+1}$ satisfy condition (PNV) and $\hat{\theta}$ satisfies equation (A.1).

\section{Log-concavity}

Lemma A.1 (MLRP) If $f$ is log-concave then it satisfies the monotone likelihood ratio property - for any $s_{1}>s_{2}$,

$$
\frac{p\left(s_{1} \mid \theta\right)}{p\left(s_{2} \mid \theta\right)}=\frac{f\left(\sqrt{\tau}\left(s_{1}-\theta\right)\right)}{f\left(\sqrt{\tau}\left(s_{2}-\theta\right)\right)}
$$

is increasing in $\theta$.

\section{PROOF:}

Log-concavity of $f$ means $\frac{f^{\prime}}{f}$ is decreasing. Differentiating the above expression with respect to $\theta$ and using the fact that $\frac{f^{\prime}}{f}$ is decreasing, we get the above result.

Lemma A.2 If $f$ is log-concave, then for $A>B \geq 0, P(\theta \geq A \mid s, \theta \geq B)$ is (weakly) increasing in $s$ for all $s \in\left[-\frac{\sigma}{2}, 1+\frac{\sigma}{2}\right]$.

\section{PROOF:}

For any $s \geq A+\frac{\sigma}{2}$, this probability is 1 and thus the weak monotonicity always holds. Let us consider any $s \in\left[-\frac{\sigma}{2}, A+\frac{\sigma}{2}\right)$,

$$
P(\theta \geq A \mid s, \theta \geq B)=\frac{\int_{A}^{s+\frac{\sigma}{2}} \pi(\theta) f(\sqrt{\tau}(s-\theta)) d \theta}{\int_{\max \left\{s-\frac{\sigma}{2}, B\right\}}^{s+\frac{\sigma}{2}} \pi(\theta) f(\sqrt{\tau}(s-\theta)) d \theta}=\frac{1}{1+\frac{\int_{\max \left\{s-\frac{\sigma}{2}, B\right\}}^{A} \pi(\theta) f(\sqrt{\tau}(s-\theta)) d \theta}{\int_{A}^{s+\frac{\sigma}{2}} \pi(\theta) f(\sqrt{\tau}(s-\theta)) d \theta}}
$$

Consider any $s_{1}$ and $s_{2}$ such that $-\frac{\sigma}{2} \leq s_{2}<s_{1}<A+\frac{\sigma}{2}$, we want to show that

$$
P\left(\theta \geq A \mid s_{1}, \theta \geq B\right)>P\left(\theta \geq A \mid s_{2}, \theta \geq B\right)
$$

First of all, since $f\left(\sqrt{\tau}\left(s_{1}-\theta\right)\right)=0$ for all $\theta \in\left[s_{2}-\frac{\sigma}{2}, s_{1}-\frac{\sigma}{2}\right)$,

$$
\int_{\max \left\{s_{1}-\frac{\sigma}{2}, B\right\}}^{A} \pi(\theta) f\left(\sqrt{\tau}\left(s_{1}-\theta\right)\right) d \theta=\int_{\max \left\{s_{2}-\frac{\sigma}{2}, B\right\}}^{A} \pi(\theta) f\left(\sqrt{\tau}\left(s_{1}-\theta\right)\right) d \theta .
$$


Moreover, based on Lemma A.1,

$$
\begin{aligned}
& \int_{\max \left\{s_{2}-\frac{\sigma}{2}, B\right\}}^{A} \pi(\theta) f\left(\sqrt{\tau}\left(s_{1}-\theta\right)\right) d \theta \\
= & \int_{\max \left\{s_{2}-\frac{\sigma}{2}, B\right\}}^{A} \pi(\theta) f\left(\sqrt{\tau}\left(s_{2}-\theta\right)\right)\left(\frac{f\left(\sqrt{\tau}\left(s_{1}-\theta\right)\right)}{f\left(\sqrt{\tau}\left(s_{2}-\theta\right)\right)}\right) d \theta \\
< & \left(\frac{f\left(\sqrt{\tau}\left(s_{1}-A\right)\right)}{f\left(\sqrt{\tau}\left(s_{2}-A\right)\right)}\right) \cdot \int_{\max \left\{s_{2}-\frac{\sigma}{2}, B\right\}}^{A} \pi(\theta) f\left(\sqrt{\tau}\left(s_{2}-\theta\right)\right) d \theta .
\end{aligned}
$$

On the other hand, since $s_{1}+\frac{\sigma}{2}>s_{2}+\frac{\sigma}{2}$, we have

$$
\int_{A}^{s_{1}+\frac{\sigma}{2}} \pi(\theta) f\left(\sqrt{\tau}\left(s_{1}-\theta\right)\right) d \theta \geq \int_{A}^{s_{2}+\frac{\sigma}{2}} \pi(\theta) f\left(\sqrt{\tau}\left(s_{1}-\theta\right)\right) d \theta .
$$

Based on Lemma A.1, we have

$$
\begin{aligned}
& \int_{A}^{s_{2}+\frac{\sigma}{2}} \pi(\theta) f\left(\sqrt{\tau}\left(s_{1}-\theta\right)\right) d \theta=\int_{A}^{s_{2}+\frac{\sigma}{2}} \pi(\theta) f\left(\sqrt{\tau}\left(s_{2}-\theta\right)\right)\left(\frac{f\left(\sqrt{\tau}\left(s_{1}-\theta\right)\right)}{f\left(\sqrt{\tau}\left(s_{2}-\theta\right)\right)}\right) d \theta \\
> & \left(\frac{f\left(\sqrt{\tau}\left(s_{1}-A\right)\right)}{f\left(\sqrt{\tau}\left(s_{2}-A\right)\right)}\right) \cdot \int_{A}^{s_{2}+\frac{\sigma}{2}} \pi(\theta) f\left(\sqrt{\tau}\left(s_{2}-\theta\right)\right) d \theta .
\end{aligned}
$$

The above inequalities implies that

$$
\frac{\int_{\max \left\{s_{1}-\frac{\sigma}{2}, B\right\}}^{A} \pi(\theta) f\left(\sqrt{\tau}\left(s_{1}-\theta\right)\right) d \theta}{\int_{A}^{s_{1}+\frac{\sigma}{2}} \pi(\theta) f\left(\sqrt{\tau}\left(s_{1}-\theta\right)\right) d \theta}<\frac{\int_{\max \left\{s_{2}-\frac{\sigma}{2}, B\right\}}^{A} \pi(\theta) f\left(\sqrt{\tau}\left(s_{2}-\theta\right)\right) d \theta}{\int_{A}^{s_{2}+\frac{\sigma}{2}} \pi(\theta) f\left(\sqrt{\tau}\left(s_{2}-\theta\right)\right) d \theta},
$$

and thus, for any $-\frac{\sigma}{2}<s_{2}<s_{1}<A+\frac{\sigma}{2}$,

$$
P\left(\theta \geq A \mid s_{1}, \theta \geq B\right)>P\left(\theta \geq A \mid s_{2}, \theta \geq B\right) .
$$

\section{Violation of Acharya and Ramsay (2013) sufficient condition}

Acharya and Ramsay (2013) (henceforth AR) consider a global game of regime change game with one-sided dominance. The model in AR can be mapped to our setting by interpreting Cooperate as Attacking $\left(a_{i}=1\right)$ and Defect as Not Attacking $\left(a_{i}=0\right)$. In AR, there is no dominance region where an agent will Cooperate regardless of the opponent's action. Hence, it is similar with PNV which eliminates the dominance region of attacking. Also, in AR, a lower signal will encourage the agents to Defect while in our setting a higher signal encourages 
agents to Not attack. Hence, the actual inequality in Condition (AR) below is reverse in their paper. Finally, the payoff specification is such that their $\frac{b_{i}}{b_{i}+w_{i}}=p$ in out setting.

Mapping AR's Assumption A1(ii) to our model, we have the following condition. For any $\hat{s}$,

$$
\mathbb{P}\left(s_{-i} \geq \hat{s} \mid s_{i}=\hat{s}, \theta \geq 0\right)>p
$$

which guarantees that no attack is the unique cutoff equilibrium when there are two players. The intuition is simple. Let us define the set of private signal for which an agent may attack -

$$
A_{i}=\left\{s_{i} \mid \text { Attacking }\left(a_{i}\left(s_{i}\right)=1\right) \text { is not eliminated as never best response }\right\} .
$$

Take $\hat{s}=\sup _{i} \cup A_{i}$. Then for any agent receiving private information $s>\hat{s}$ will not attack because attacking is eliminated as a dominated strategy. Think about any agent with private information $\hat{s}$. Given Condition (AR), the probability that the other agent receives a signal higher than $\hat{s}$ and thus does not attack is higher than $p$. Thus, not attacking is strictly preferred, which violates the definition of $\hat{s}$. That is why no attacking is the only possible equilibrium.

We take the standard global game information structure and assume that $F$ is log-concave. We did not assume the Condition (AR). In our setting, what we need is that for any $\hat{s}$,

(CI) $\mathbb{P}$ (regime survives when agents follow cutoff strategy $\left.\hat{s} \mid s_{i}=\hat{s}, \theta \geq 0\right)>p$,

We argue in lemma 1 that when $\alpha$ is small enough, the above inequality (CI) holds. However, as shown below, condition (AR) may not hold true under the assumption of small group size. For simplicity, let us assume that the prior is uniform.

Lemma A.3 For any $\alpha$ and $p>1 / 2$, there exists $\hat{s}<\alpha+\sigma / 2$ such that

$$
\mathbb{P}\left(s_{-i} \geq \hat{s} \mid s_{i}=\hat{s}, \theta \geq 0\right) \leq p .
$$

PROOF:

$$
\mathbb{P}\left(s_{-i} \geq \hat{s} \mid s_{i}=\hat{s}, \theta \geq 0\right)=\int_{\max \{0, \hat{s}-\sigma / 2\}}^{\hat{s}+\sigma / 2} \mathbb{P}\left(s_{-i} \geq \hat{s} \mid \theta\right) d \mathbb{P}\left(\theta \mid s_{i}=\hat{s}, \theta \geq 0\right)
$$


Given uniform prior, this simplifies to

$$
\begin{aligned}
& =\int_{\max \{0, \hat{s}-\sigma / 2\}}^{\hat{s}+\sigma / 2}(1-F(\sqrt{\tau}(\hat{s}-\theta))) \cdot d\left(\frac{1-F(\sqrt{\tau}(\hat{s}-\theta))}{F(\sqrt{\tau} \hat{s})}\right) \\
& =\frac{1}{2 F(\sqrt{\tau} \hat{s})} \cdot\left[(1-F(\sqrt{\tau}(\hat{s}-\theta)))^{2}\right]_{\max \{0, \hat{s}-\sigma / 2\}}^{\hat{s}+\sigma / 2} \\
& =\left\{\begin{array}{l}
\frac{1}{2}, \text { for } \hat{s} \in[\sigma / 2,1+\sigma / 2] \\
1-\frac{F(\sqrt{\tau} \hat{s})}{2} \text { for } \hat{s} \in[-\sigma / 2, \sigma / 2]
\end{array}\right.
\end{aligned}
$$

When $p>1 / 2$, there is some $\hat{s} \in[\sigma / 2, \alpha+\sigma / 2]$ for which condition (AR) is violated. ${ }^{1}$ It holds true only when $\hat{s}<\sigma F^{-1}(2(1-p))$.

In the absence of viability test, an agent believes that half of the agents have received higher signal than him and the other half has received lower signals than him. PNV does not change this belief uniformly. For an agent who receives a low private signal, PNV convinces him that there is a larger fraction of other agents who receive higher signals than him. For example, consider the extreme case - an agent who receives signal $s_{i}=-\sigma / 2$. Upon receiving the PNV, he understands that $\theta=0$ and all other agents will receive higher private signals. On the other hand, PNV will not have an impact on the belief of an agent with private signal $s_{i} \geq \sigma / 2$ regarding other agents' signals. He always believes that $1 / 2$ mass of agents have received higher signal. Thus, the Condition (AR) is restrictive and does not hold for all $\hat{s}$ when $p>1 / 2$.

It is important to point out that a small group size $\alpha$ does not affect agent $i$ 's belief about the private signal of agent $j$. As discussed in Section II, a small group size $\alpha$ reduces the survival criteria. The combined effect of a sufficiently small group size and the PNV makes condition (CI) satisfied.

\section{Time varying fundamental}

In our model, nature chooses the fundamental $\theta$ at time 0 and $\theta$ can only decreases from time 0 to 1 if there are endogenous attacks. We show that sufficient diffusion can dissuade the agents from attacking. In practice, the fundamental $\theta$ can vary over time. In particular, if the agents are worried that the regime may not survive because of some negative shocks in future, then persuading them not to attack will be more difficult.

For simplicity, we work with uniform prior. Let $Z_{t}$ be the number of negative shocks that can arrive by time $t$. $Z_{t}$ follows a Poisson arrival process with

\footnotetext{
${ }^{1}$ Note that if agents receive private information $s \geq \alpha+\sigma / 2$, he understands that $\theta \geq \alpha$ and thus the regime succeeds even if all agents (with measure $\alpha$ ) attack. In this case, the condition $\bar{A} R$ is redundant.
} 
parameter $\lambda$ - i.e.,

$$
\mathbb{P}\left(Z_{t}=n\right)=\frac{(\lambda t)^{n}}{n !} e^{-\lambda t}
$$

where $\lambda$ is the arrival rate of shock. We assume that $\lambda$ is sufficiently small.

Assumption A.1 $e^{-\lambda}>p$.

For simplicity, we further assume that the shocks are large enough in size in the sense that once a shock hits, the regime cannot survive no matter what agents do. This means that when the regime passes the $j$ th viability test, the agents know that a shock has not arrived yet. Note that, given these assumptions, if no agent attacks a viable regime from time 0 to 1 , the probability the regime will survive is greater than $p$. In other words, if Assumption A.1 is violated, even if all agents decided not to attack, the probability that a viable regime will succeed is lower than $p$, independent of the realization of $\theta$. Hence, attacking is the dominant action, regardless of agents' information about the underlying fundamental $\theta$ and past actions. Thus, there is no room for persuasion. Below, we show that viability tests with sufficient frequency could persuade the agents not to attack under these assumptions (whenever there is some room for persuasion).

Claim A.1 Under Assumption A.1, there exists $\alpha^{*}\left(e^{\lambda} p, \tau\right)>0$ such that a diffused policy $J>\frac{1}{\alpha^{*}\left(e^{\lambda} p, \tau\right)}$ is persuasive.

\section{PROOF:}

We will show the induction statement $N_{j}$ holds true as in Lemma 3 . Then following the same inductive steps as in Theorem 1 we can arrive at the result.

For any group $j$, the probability that no shock will arrive until 1 is

$$
\mathbb{P}\left(Z_{1}=Z_{(j-1) \alpha}\right)=\mathbb{P}\left(Z_{1}-Z_{(j-1) \alpha}=0\right)=\mathbb{P}\left(Z_{1-(j-1) \alpha}=0\right)=e^{-\lambda(1-(j-1) \alpha))} .
$$

Consider the agent in group $j$ who receives the cutoff signal $\hat{s}_{j}$ (as in Lemma 3 ). As before, he believes that the regime passes the $j$ th viability test if $\theta \geq \underline{\theta}_{j-1}$ and it will survive the attacks from group $j$ if $\theta \geq A_{j}\left(\hat{s}_{j}, \alpha, \underline{\theta}_{j-1}\right)\left(A_{j}\right.$ is defined as in Condition $\left.\left(A_{j}^{\alpha}\right)\right)$. The statement $M_{j}$ is the same as in the paper. Under $M_{j}$, no one in group $j^{\prime}>j$ attacks a regime that passes the $j+1$ th viability test (independent of the cutoff strategies played by agents in early groups), which means a viable regime can only fail if the negative shock arrives after time $j \alpha$.

Given $M_{j}$ holds true, this means that if $\theta \geq A_{j}\left(\hat{s}_{j}, \alpha, \underline{\theta}_{j-1}\right)$, then in the absence of any future shock, the regime will succeed. Therefore, after receiving PNV, he believes that the regime will succeed with probability at least

$$
\begin{gathered}
\mathbb{P}(\text { No future shock }) \cdot \frac{\left.\mathbb{P}\left(\theta \geq A_{j}\left(\hat{s}_{j}, \alpha, \underline{\theta}_{j-1}\right)\right) \mid \hat{s}_{j}, \text { No future shock }\right)}{\mathbb{P}\left(\theta \geq \underline{\theta}_{j-1} \mid \hat{s}_{j}, \text { No future shock }\right)} \\
=e^{-\lambda(1-(j-1) \alpha))} \cdot \frac{F\left(\sqrt{\tau}\left(\hat{s}_{j}-A_{j}\right)\right)}{F\left(\sqrt{\tau}\left(\hat{s}_{j}-\underline{\theta}_{j-1}\right)\right)} .
\end{gathered}
$$


Substituting the Aggregate Condition, we get

$$
\begin{gathered}
e^{-\lambda(1-(j-1) \alpha))} \cdot \frac{\left(\frac{A_{j}-\underline{\theta}_{j-1}}{\alpha}\right)}{F\left(F^{-1}\left(\frac{A_{j}-\underline{\theta}_{j-1}}{\alpha}\right)+\alpha \sqrt{\tau}\left(\frac{A_{j}-\underline{\theta}_{j-1}}{\alpha}\right)\right)} \\
=e^{-\lambda(1-(j-1) \alpha))} \cdot G^{u}\left(\left(\frac{A_{j}-\underline{\theta}_{j-1}}{\alpha}\right), \alpha\right)
\end{gathered}
$$

If $\alpha<\alpha^{*}\left(e^{\lambda} p, \tau\right)$, then $G^{u}(x, \alpha)>e^{\lambda} p$ for all $x \in[0,1]$. Therefore, the agent who receives the cutoff signal $\hat{s}_{j}$ believes that the regime will succeed with probability strictly higher than

$$
e^{-\lambda(1-(j-1) \alpha))} \cdot e^{\lambda} p=e^{(j-1) \alpha} p \geq p .
$$

Therefore, the marginal agent who receives the cutoff signal strictly prefers not attacking. This implies that there is no cutoff equilibrium in which an agent in group $j$ will attack the regime that passes the $j$ th viability test. Given $M_{J}$ is true, it follows that no agent will attack a viable regime as in Theorem 1.

Due to the exogenous negative shocks, the regime could fail with a higher probability. However, this claim shows that, if the probability of having such shock is small, our diffused policy could still guarantee that, for any regime that passes the viability test, there is no endogenous attack against a viable regime.

\section{Arrival of new information}

In our baseline model, we assume that the agents move sequentially, but they do not know the past actions by other agents. Agents have some private information about the underlying fundamental $\theta$. It is the principal who dynamically discloses some partial information based on the fundamental and the past attacks, to dissuade the agents from attacking.

However, in practice, the agents may get some new private signals on their own, while the principal runs the viability tests. This may make it harder to persuade the agents to ignore their private information and follow the principal's recommendation. Below we investigate whether sufficient diffusion will still be persuasive.

To keep it analytically tractable, we assume that the agents have improper prior over $\theta$ and the noise $\epsilon$ follows a standard normal distribution. This satisfies log-concavity, but the support is not bounded as we assumed in the main paper. This assumption is conventional in the global game literature (See Morris and Shin (2003) or Angeletos, Hellwig and Pavan (2006)). Under these two assumptions, by replacing the $F$ by $\Phi$ in the definition of $G$ (see equation $(\mathrm{G})$ ), we can define $\alpha_{\Phi}^{*}(p, \tau)$ and prove the main result as we did in the paper. 
Recall the diffused policy can work as long as $J>\frac{1}{\alpha_{\Phi}^{*}(p, \tau)}$, in which the critical group size $\alpha_{\Phi}^{*}$ depends on the precision of private information $\tau$. As we have shown in the comparative statics in the discussion section, $\alpha_{\Phi}^{*}$ decreases with $\tau$. If new noisy private signals arrive over time, then the agents who move later will be more informed about $\theta$. With normal distribution of noises, the precision of private information that consists of multiple pieces of noisy signals is just the summation of the precision of each signal. If the arrival of new information is independent of the diffused policy, then the principal can design the policy targeting at the most informed agent, who has the highest precision of private information $\tau_{\max }$. A diffused policy with $J>\frac{1}{\alpha_{\Phi}^{*}\left(p, \tau_{\max }\right)}$ is persuasive.

The above argument is not necessarily true when the precision of the best informed agent's private information depends on the policy $J$. Below, we consider a simple example and show that it will be more difficult to persuade the agents if they become better informed about the underlying fundamental $\theta$ when the policy becomes more diffused. Nevertheless, they can be persuaded.

Precision increases with diffusion.- Suppose that the principal adopts a diffused policy $J$. Then, when the principal runs the $j$ th viability test, for any $j>1$, each agent $i$ receives an additional noisy private signal

$$
s_{i}^{j}=\theta+\sigma \epsilon_{i}^{j},
$$

where $\epsilon_{i}^{j} \sim N(0,1)$. Note that the first viability test does not disclose anything new about the past attacks. We use the convention $s_{i}^{1}=s_{i}$, where $s_{i}$ is the initial noisy private signal as in our baseline model.

Thus, the agents in group $j$ receive $j$ different noisy private signals. Given the improper prior and Normal error, the updated belief of an agent $i$ in group $j$ is

$$
\theta \mid\left\{s_{i}^{l}\right\}_{l=1}^{j} \sim N\left(\frac{\sum_{l=1}^{j} \tau s_{i}^{l}}{\sum_{l=1}^{j} \tau}, \frac{1}{\sum_{l=1}^{j} \tau}\right)=N\left(\frac{1}{j} \sum_{l=1}^{j} s_{i}^{l}, \frac{1}{j \tau}\right) .
$$

This shows that that the later groups are better informed about $\theta$. More importantly, as the policy becomes more diffused ( $J$ increases and $\alpha$ decreases), the precision of this private information can become as high as $J \tau=\frac{\tau}{\alpha}$.

For a policy to be persuasive without new arrival of private signals, we need $\alpha<$ $\alpha_{\Phi}^{*}(p, \tau)$. Hence, it is possible that $\alpha>\alpha_{\Phi}^{*}\left(p, \frac{\tau}{\alpha}\right)$, which means, with new arrivals of private signals, the group size may not be small enough to make $G(x, \alpha)>p$ for all $x \in[0,1]$. Thus, this type of diffused policy may no longer be persuasive when agents have additional private information. But is this true for any $\alpha$ ? If so, then our main result will be violated.

In the above simple example, we can show there exists $\check{\alpha}(p, \tau)$ such that a diffused policy with $J>\frac{1}{\check{\alpha}}$ is persuasive.

To understand this, let us consider the last group who is the best informed 
one. Agents in group $J$ have private information with precision $\frac{\tau}{\alpha}$. Following the arguments in the proof of Lemma 3, we need the group size $\alpha$ to be sufficiently small so that the agent in group $J$ who receives the cutoff signal believes that

$$
\min _{x \in[0,1]} \frac{x}{\Phi\left(\Phi^{-1}(x)+\sqrt{\alpha} \sqrt{\tau} x\right)}>p
$$

Recall that $x=\frac{A_{J}-\underline{\theta}_{J-1}}{\alpha} \in[0,1]$. The above probability is still decreasing in $\alpha$. Hence, it is easy to see that whenever $\alpha<\left(\alpha_{\Phi}^{*}(p, \tau)\right)^{2}$,

$$
\min _{x \in[0,1]} \frac{x}{\Phi\left(\Phi^{-1}(x)+\sqrt{\alpha} \sqrt{\tau} x\right)}>\min _{x \in[0,1]} \frac{x}{\Phi\left(\Phi^{-1}(x)+\alpha^{*} \sqrt{\tau} x\right)}=p .
$$

Therefore, there exists $\check{\alpha}=\left(\alpha_{\Phi}^{*}\right)^{2}$ such that for any $\alpha<\check{\alpha}$, argument $N_{J}$ is true. For $j<J$, since the precision is smaller, $\alpha<\check{\alpha}$ can guarantee $N_{j}$ is true. Hence, we can apply the inductive argument from $M_{J}$ and show that the diffused policy $J>\frac{1}{\check{\alpha}}$ is persuasive.

In this example, when $J$ increases, the precision of accumulated information $J \tau$ also increases at a rate proportional to $J$. One can see from the proof that as long as the precision of the accumulated private information is growing at a rate lower than $J^{2}$ (or $O\left(J^{2}\right)$ ), a sufficiently diffused policy (or a sufficiently high $J$ ) can still be persuasive.

When the precision of private signal increases at a sufficiently high rate as more diffused policy is adopted, then no matter how frequent the principal runs the viability tests, the PNV cannot be effective enough to overcome the effect of more precise private information. Below, we use a simple example to confirm this logic.

A Counter Example.- Consider the case in which the agents not only receive additional information, but the additional information becomes more precise over time. Let us assume that $\tau>1$ and the $j$ th private signal precision is $\tau^{j}$.

$$
s_{i}^{j}=\theta+\frac{1}{\sqrt{\tau^{j}}} \epsilon_{i}^{j},
$$

Then the updated belief

$$
\begin{gathered}
\theta \mid\left\{s_{i}^{l}\right\}_{l=1}^{j} \sim N\left(\frac{\sum_{l=1}^{j} \tau^{l} s_{i}^{l}}{\sum_{l=1}^{j} \tau^{l}}, \frac{1}{\sum_{l=1}^{j} \tau^{l}}\right)=N\left(\xi_{i}^{j}, \frac{1}{\hat{\tau}^{j}}\right), \\
\text { where } \hat{\tau}^{j} \equiv \sum_{l=1}^{j} \tau^{l}=\frac{\tau^{j+1}-\tau}{\tau-1}, \text { and } \xi_{i}^{j} \equiv \sum_{l=1}^{j} \frac{\tau^{j}}{\hat{\tau}^{j}} s_{i}^{j} .
\end{gathered}
$$

Following the same steps as in the previous example, we can say that agent in group $J$ who receives the cutoff signal believes that the regime will succeed with 
probability

$$
\frac{x}{\Phi\left(\Phi^{-1}(x)+\alpha \sqrt{\hat{\tau}^{J}} x\right)} .
$$

It is easy to check that $\alpha^{2} \hat{\tau}^{J}=\frac{\tau}{\tau-1} \frac{\tau^{J}-1}{J^{2}}$ is increasing in $J$ when $J$ is sufficiently large (or $J>\frac{2}{\ln \tau}$ ).

Hence, unlike in the previous example, when $J$ increases and thus $\alpha$ decreases, the marginal agent becomes more pessimistic about the success of the regime. Thus, for any critical group size $\check{\alpha}$, we can find $\alpha<\check{\alpha}$ such that there exist some cutoff equilibrium in which the marginal agent believes the regime will succeed with probability $p$. Accordingly, one can construct a cutoff equilibrium with positive attack against a viable regime. Hence, the arrival of new information in the dynamic setting may break our result. Our result is only robust to the case in which the new arrival of information (induced by a more diffused policy) would not increase the precision of agents' private information very rapidly.

\section{REFERENCES}

Acharya, Avidit, and Kristopher W Ramsay. 2013. "The calculus of the security dilemma." Quarterly Journal of Political Science, 8(2): 183-203.

Angeletos, George-Marios, Christian Hellwig, and Alessandro Pavan. 2006. "Signaling in a Global Game: Coordination and Policy Traps." Journal of Political Economy, 114(3): 452-484.

Goldstein, Itay, and Chong Huang. 2016. "Bayesian Persuasion in Coordination Games." The American Economic Review, 106(5): 592-596.

Inostroza, Nicolas, and Alessandro Pavan. 2017. "Persuasion in Global Games with Application to Stress Testing." http://faculty.wcas . northwestern.edu/ apa522/persuasion-GG.pdf.

Morris, Stephen, and Hyun Song Shin. 2003. "Global Games: Theory and Applications." In Advances in Economics and Econometrics (Proceeding of the Eighth World Congress of the Econometric Society). , ed. Dewatripont, Hansen and Turnovsky. Cambridge University Press. 